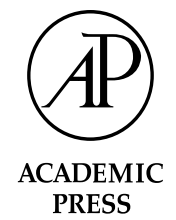

Available online at www.sciencedirect.com

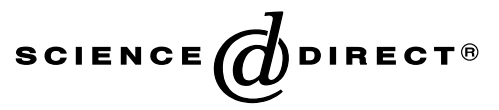

Consciousness and Cognition 12 (2003) 231-256

Consciousness

and

Cognition

www.elsevier.com/locate/concog

\title{
Functional neuroanatomy of altered states of consciousness: The transient hypofrontality hypothesis
}

\author{
Arne Dietrich* \\ Department of Psychology, Behavioral Neuroscience Laboratory, \\ Georgia College and State University, Milledgeville, GA 31061, USA
}

Received 1 April 2002

\begin{abstract}
It is the central hypothesis of this paper that the mental states commonly referred to as altered states of consciousness are principally due to transient prefrontal cortex deregulation. Supportive evidence from psychological and neuroscientific studies of dreaming, endurance running, meditation, daydreaming, hypnosis, and various druginduced states is presented and integrated. It is proposed that transient hypofrontality is the unifying feature of all altered states and that the phenomenological uniqueness of each state is the result of the differential viability of various frontal circuits. Using an evolutionary approach, consciousness is conceptualized as hierarchically ordered cognitive function. Higher-order structures perform increasingly integrative functions and thus contribute more sophisticated content. Although this implies a holistic approach to consciousness, such a functional hierarchy localizes the most sophisticated layers of consciousness in the zenithal higher-order structure: the prefrontal cortex. The hallmark of altered states of consciousness is the subtle modification of behavioral and cognitive functions that are typically ascribed to the prefrontal cortex. The theoretical framework presented yields a number of testable hypotheses.

(C) 2002 Elsevier Science (USA). All rights reserved.
\end{abstract}

Keywords: Altered states; Consciousness; Prefrontal cortex; Philosophy of mind; Dreaming; Runner's high; Meditation; Hypnosis; Daydreaming; Drugs

\footnotetext{
*Fax: 1-912-445-0856.

E-mail address: adietric@mail.gcsu.edu.
} 


\section{The hierarchy of consciousness}

Modern brain research conceptualizes cognitive function as hierarchically ordered. Evolutionary pressures forced the development of ever more integrative neural structures able to process increasingly complex information. This in turn led to increased behavioral flexibility and adaptability. The cerebral cortex, and in particular the prefrontal cortex, is at the top of that hierarchy, representing the neural basis of higher cognitive functions (e.g., Frith \& Dolan, 1996; Fuster, 2000). Historically, consciousness was approached in a similar manner (for a review, see Markowitsch, 1995). Consciousness was defined by selecting various attributes, such as self-reflective consciousness, attention, memory, perception, and arousal, which were ordered in a functional hierarchy with the frontal lobe necessary for the 'top' attributes. Recent theories of the neural correlates of consciousness similarly emphasize frontal cortex function for higher attributes (e.g., Crick \& Koch, 1998; Dehaene \& Naccache, 2001).

Perhaps the single most important functional division in the neocortex is the central sulcus. It demarcates the frontal lobe from the three posterior cortices, the temporal, the occipital, and the parietal, which will be collectively referred to as TOP. Functions of TOP are very different from those of the frontal lobe. TOP neurons are devoted primarily to perception, learning, and memory. The primary sensory cortices of all sense modalities are located in TOP and its association cortex further assembles and assimilates sensory information decoded initially in primary cortex. It is generally agreed that TOP is the site of long-term memory storage (e.g., Gilbert, 2001). The frontal lobe, located rostal to the central sulcus, neither receives direct sensory input nor stores long-term memory. The prefrontal cortex, which comprises approximately half of the frontal lobe in humans, is involved in executive functions. It integrates perceptual information, formulates plans and strategies for appropriate behavior in a given situation, and instructs the adjacent motor cortices to execute its computational product.

At the outset, it should be made clear that it is not suggested that the prefrontal cortex is the seat of consciousness. Rather, the prefrontal cortex enables the top layers of consciousness by contributing the highest-order cognitive functions to the conscious experience. A large body of evidence from lesion, imaging, and electrophysiological studies suggests that the prefrontal cortex, in contrast to various brainstem nuclei or the intralaminar nuclei, is not necessary for basic awareness (e.g., Baars, 1995; Bogen, 1995). Likewise, evidence suggests that initial and much ensuing information processing on perception, attention, or memory occurs in other brain areas before further integration in the frontal lobes (e.g., Crick \& Koch, 1998; Pollen, 1999; Taylor, 2001). Indeed, information processing on these cognitive functions appears to be all but complete so that frontal lobe patients do not show any evidence of dysfunction when tested for them, either directly or with conventional intelligence tests (Brickner, 1936; Hebb, 1939). Rather than adding further detail to the analysis, the frontal cortex utilizes this highly processed information to enable still higher cognitive functions such as a self-construct (Keenan, Wheeler, Gallup, \& Pascual-Leone, 2000; Vogeley, Kurthen, Falkai, \& Maier, 1999), self-reflective consciousness (Courtney, Petit, Haxby, \& Ungerleider, 1998; Vogeley et al., 
2001), complex social function (Damasio, 1994), abstract thinking (e.g., Rylander, 1948), cognitive flexibility (Lhermitte, 1983; Lhermitte, Pillon, \& Serdaru, 1986), planning (Norman \& Shallice, 1986; Shallice \& Burgess, 1991), willed action (Frith \& Dolan, 1996), and theory of mind (Frith \& Frith, 2001; Povinelli \& Preuss, 1995; Stone, Baron-Cohen, \& Knight, 1998).

Three other cognitive functions of the prefrontal cortex, working memory (Fuster, 2000; Goldman-Rakic, 1992) temporal integration (e.g., Fuster, 1995; Knight \& Grabowecky, 1999; Kolb, 1984), and sustained and directed attention (e.g., Posner, 1994; Sarter, Givens, \& Bruno, 2001) provide the infrastructure to compute these complex cognitive functions by providing a buffer to hold information in mind and order it in space-time (Dehaene \& Naccache, 2001; Duncan \& Owen, 2000). It is this superimposing of already highly complex mental constructs that dramatically increases cognitive flexibility and permits a unified phenomenological experience.

The prefrontal cortex is not a single unit. It is functionally divided into ventromedial (VM) and dorsolateral (DL) aspects. Lesion and functional imaging studies have shown that different prefrontal circuits, along with their respective cortical and subcortical connections, are implicated in specific cognitive abilities. There is also a discernible hemispheric specialization, further suggesting that these cognitive processes are not global functions of the frontal lobe. For instance, semantic memory retrieval is implemented in the left DL, whereas activation of the right DL is most closely associated with sustained attention (for a review, see Cabeza \& Nyberg, 2000; Duncan \& Owen, 2000).

The most common deficit associated with the VM region of the prefrontal cortex is impaired social function. This deficit was epitomized by the famous case of Phineas Gage, who after a freak accident that damaged his frontal lobe, exhibited what is now recognized as the typical frontal syndrome: inappropriate social behaviors, lack of moral judgment, few social inhibitions, few abstract thought processes, an inability to plan for the future, and/or difficulty to maintain a plan of action. He showed a lack of concern for himself and others and behaved with little regard for social constraints. Damasio (1994) suggested that the VM, with its intricate connections to the limbic system, might assess the personal consequences of one's behavior, and that the resulting emotions are an essential prerequisite to making logical and rational decisions.

Damage to the DL prefrontal cortex does not involve changes in personality and emotion. The DL does not receive direct innervation from subcortical structures such as the amygdala that are involved in affective behavior (Petrides \& Pandya, 1999). Its primary input comes from TOP and its primary output is the motor cortices. Primarily, research has implicated the DL in working memory, directed attention, and temporal integration (Fuster, 2000; Goldman-Rakic, 1992; Knight \& Grabowecky, 1999; Posner, 1994). With its ability to sustain online processing in real time, the working memory buffer appears to be a prerequisite for cognitive flexibility, abstract thinking, strategic planning, access to memory, as well as sentience. It has been suggested that it is in these DL circuits that full-fledged, self-reflective consciousness comes about (e.g., Baddeley, 2000; Courtney et al., 1998; Dehaene \& Naccache, 2001; Posner, 1994). 
Perseverance, or the inability to shift between modes of thinking, is the most reliable deficit associated with damage to the DL prefrontal cortex (Brauer Boone, 1999). Perseverance is perhaps most indicative of a lack of cognitive flexibility and ability to think abstractly. It is commonly tested for and quantified with the Wisconsin Card Sorting Task (WCST), in which cards are sorted by one of three characteristics: color, number, or shape. The patient is required to discover the sorting rule empirically using only feedback from the examiner about their selection. When the examiner changes the sorting rule patients are required to adapt to the new rule. Patients with DL damage show perseverance errors in this task. They keep sorting according to the old rule even in the face of obvious discrepancy. This is frequently interpreted as a failure of working memory as relevant past behavior is required to perform the task successfully. A comparable deficit can be produced in rats. Using a T-maze, a rat is trained over many trials to find food located in one of the T's arms. Once the rule (right or left) is acquired according to some criterion, it is changed to the other arm. After a number of reversals, a strategy emerges that is called win/stay loose/shift, or in other words, the animal acquires the concept of shifting. Prefrontal lesions cause animals to persevere and adhere to the previously learned rule (e.g., Dietrich, Taylor, \& Passmore, 2001).

It appears that the prefrontal cortex exerts inhibitory control over inappropriate or maladaptive emotional and cognitive behaviors. Lhermitte (1983) and Lhermitte et al. (1986) documented this tendency by showing that frontal lobe patients are overly dependent on immediate cues. They tend to act on what they see without taking into account the 'bigger picture.' Similarly, patients show a strong tendency to imitate inappropriate behaviors modeled by others. As Lhermitte put it: "the sight of the movement is perceived in the patient's mind as an order to imitate; the sight of an object implies the order to use it" (p. 330). Without a fully functional frontal lobe, the patient can utilize only immediate cues and fails to select behaviors based on more universal principles. Thus, the frontal lobe provides for cognitive flexibility and freedom and releases us from the slavery of direct environmental triggers or the memory stored in TOP.

The picture presented thus far is that of a hierarchy of layers of consciousness that is intimately associated with the functional hierarchical organization of the brain. This view assumes that full-fledged consciousness is a global function of a fully operational brain. The concept of a functional hierarchy of consciousness can be incorporated into the larger framework of global workspace theory (Baars, 1989; Dehaene \& Naccache, 2001). Any pattern of activation that constitutes a given transient global workspace can be conceptualized as a functional hierarchy in which prefrontal neurons provide a higher-order computation than, for example, TOP neurons. Although this is a holistic view of consciousness, it is evident that not all areas of the brain, not even all areas of the neocortex, contribute equally to consciousness. Damage to any layer will result in an alteration of consciousness, but the lower the level of malfunctioning the more fundamental the alteration. It is no easy task to establish such a hierarchy in detail, and perhaps impossible to order the top layers as they mutually enable each other. Primarily as a visual aid, the hierarchy is conceptualized as an inverted pyramid (see Fig. 1). 


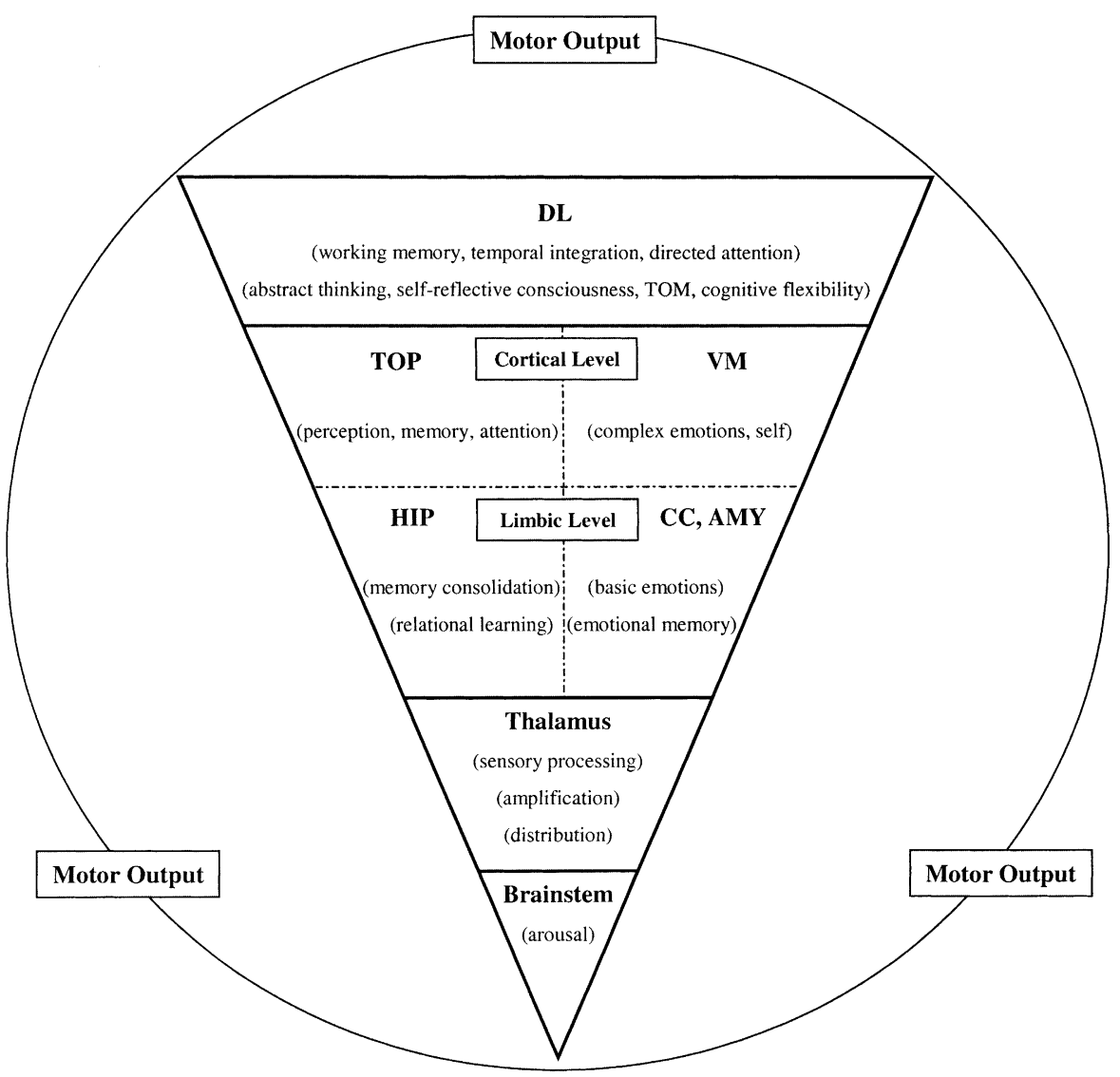

Fig. 1. The hierarchy of consciousness. In a framework that conceptualizes consciousness as hierarchically ordered cognitive function, no single neural structure is necessary and sufficient for consciousness. Yet, not all areas of the brain contribute equally to consciousness. Damage to any layer will result in an alteration of consciousness, but the lower the level of malfunctioning the more fundamental the alteration. Full-fledged consciousness requires the engagement of the zenithal higher-order structure: the dorsolateral prefrontal cortex. The concept of a functional hierarchy of consciousness can be incorporated into the larger framework of global workspace theory (Baars, 1989). Any pattern of activation that constitutes a given transient global workspace can be conceptualized as a functional hierarchy in which prefrontal neurons provide the highest-order computation. The hallmark of altered states of consciousness is the subtle modification of cognitive functions that are typically ascribed to the 'top' layers. Abbreviations: DL, dorsolateral; VM, ventromedial; TOP, temporal, occipital, and parietal cortex; HIP, hippocampal formation; CC, cingulate cortex; AMY, amygdala; and TOM, theory of mind.

As with global workspace theory, in such a conceptual framework, no single neural structure is necessary and sufficient for consciousness. Damage to the lowest levels, such as the mesencephalic reticular formation that is necessary for basic arousal, wipes out consciousness altogether. The highest level at which such fun- 
damental change can occur is apparently the thalamus (Bogen, 1995). Nonetheless, the thalamus might better be conceptualized as representing the next level by providing aspects to consciousness such as binding experiences together (e.g., Llinás \& Paré, 1991), preliminary processing of perceptual information (e.g., LGN), or differential amplification of information (Crick, 1994). Its precise reciprocal connections with the neocortex and the limbic system also distribute information to the appropriate places for further processing (e.g., LeDoux, 1996). It is suggested that all stages of processing beyond the thalamus provide some form of content.

It is reasonable to assume that when patients with brain damage fail to display a particular function, such as fear or abstract thinking, that their phenomenological experience does not contain its computation. Initial processing of affective content occurs in various limbic system structures (LeDoux, 1996). Consequently, dysfunction in structures such as the amygdala changes the emotional content of consciousness profoundly. For instance, lesions to the central nucleus of the amygdala will eliminate the basic emotion of fear altogether (LeDoux, 1996). The computational product of these limbic structures is used by the next levels of affective processing represented by the cingulate cortex and the VM (Damasio, 1994). Damage or dysfunction at that level results in more subtle alterations to the emotional content of consciousness, such as the context in which fear might be triggered.

The thalamus is the starting point of a separate and parallel line of information processing that is devoid of any salient information. It is represented by another set of limbic system structures, primarily the hippocampal formation, as well as TOP cortices. These structures process perceptual information and store the computation as memory. The required level of selective attention to process the information is also supplied by these structures. Again, the higher the level at which a dysfunction occurs (e.g., association vs. primary cortex) the more selective the deficit. If the information required for a cognitive construct is not computed, it cannot be brought 'online' by TOP circuits and is thus unavailable for prefrontal modules to be included in the computation of higher cognitive functions. Consequently, it would be missing in the content of consciousness, resulting in distinct phenomenological distortions.

Damasio (1999) provided a detailed account of how a complex cognitive function such as the self-construct is built at various functional neuroanatomical levels in a hierarchy of increasing complexity. The sense of self has many subcomponents such as an experience of ownership, body-centered spatial perceptivity, long-term unity of beliefs (Vogeley et al., 1999) as well as representation in somatic, kinesthetic, and motor maps (Damasio, 1999). Damage to structures that process information lower on the hierarchy will produce severe distortions such as unilateral neglect, which results from specific right parietal damage; whereas, prefrontal dysfunction might result in lesser distortion of the self such as delusions of grandeur.

The neural substrate for the highest layers of cognitive ability is the DL. As seen in Fig. 1, further sorting these layers into an ascending order is avoided. Instead, it is proposed that they mutually enable and potentiate one another. The framework presumes that working memory, temporal integration, and focused attention are global capacities of frontal tissue that provide the computational parameters for all other higher cognitive functions implemented by the prefrontal cortex. The resulting fully flavored conscious experience is a function of computations from all structures 
comprising a given global workspace that are superimposed at the level of the prefrontal cortex to provide a unified conscious experience.

It has been suggested that the content in the buffers of working memory is the current conscious awareness (e.g., Baddeley, 2000; Courtney et al., 1998; Posner, 1994). A normal, healthy brain relies heavily on this buffered information in the frontal lobe. If information is not represented in the buffers it is unconscious to the extent that we cannot reflect or report on it. At all levels of the hierarchy, including the spinal cord, neural structures have direct access to activating the motor system. This makes sense from an evolutionary perspective as these structures developed, at the time, in order to directly affect behavior. Blindsight and amygdala-driven conditioned emotional responses are good examples of that. In either case, a structure drives motor output before, if ever, sending the information up the chain for conscious processing.

It is evident that any modification of information processing, from the sensory level to the prefrontal cortex, alters the content of consciousness. Yet, changes below the level represented by the DL do not seem to affect the maximal capacity for conscious experience (provided the arousal system is properly activated). For instance, patients with unilateral neglect, anterograde amnesia, or blindsight, to name a few, can still self-reflect - just not on the particular information that is missing. Similarly, Damasio (1994) reported the case of EVR who sustained VM damage and showed no deficit in hypothetical scenarios of complex moral and social situations. He still showed the capacity indicative of the highest layers of consciousness. The frontal syndrome remained confined to 'actual' social behavior. In contrast, damage to the DL has the tendency to diminish the maximal capacity of consciousness. With the loss of top layers of consciousness, patients show little evidence for higher cognitive functions such as abstract thinking, regardless of the information that is brought 'online' by other structures.

In such a view, the prefrontal cortex does not represent a supervisory or control system. Rather, it actively implements higher cognitive functions. It is further suggested that the prefrontal cortex does not act as an inhibitory agent of older, more primitive brain structures. The prefrontal cortex restrains output from older structures not by suppressing their computational product directly but by elaborating on it to produce more sophisticated output. If the prefrontal cortex is lost, the person simply functions on the next highest layer that remains. The structures implementing these next highest layers are not disinhibited by the loss of the prefrontal cortex. Rather, their processing is unaffected except that no more sophistication is added to their processing before a motor output occurs.

\section{Altered states of consciousness}

It is proposed in this article that altered states of consciousness are due to transient prefrontal deregulation. Six conscious states that are considered putative altered states (dreaming, the runner's high, meditation, hypnosis, daydreaming, and various drug-induced states) are briefly examined. These altered states share characteristics whose proper function are regulated by the prefrontal cortex such as time distortions, 
disinhibition from social constraints, or a change in focused attention. It is further proposed that the phenomenological uniqueness of each state is the result of the differential viability of various DL circuits. To give one example, the sense of self is reported to be lost to a higher degree in meditation than in hypnosis; whereas, the opposite is often reported for cognitive flexibility and willed action, which are absent to a higher degree in hypnosis. The neutralization of specific prefrontal contributions to consciousness has been aptly called "phenomenological subtraction" by Allan Hobson (2001). The individual in such an altered state operates on what top layers remain. In altered states that cause severe prefrontal hypofunction, such as non-lucid dreaming or various drug states, the resulting phenomenological awareness is extraordinarily bizarre. In less dramatic altered states, such as long-distance running, the change is more subtle. Finally, unlike most cases of mental illness and brain damage, altered states of consciousness can be characterized as transient in nature.

\subsection{Dreaming}

Sleep is a profound alteration of consciousness. Not surprisingly, it is instigated by neural activity in the brainstem, an area near the bottom in the hierarchy of consciousness (for a review, see Hobson, Pace-Schott, \& Stickhold, 2000). Electrophysiological, neuroimaging, and psychological data strongly suggest that REM sleep is a state phenomenologically different from NREM sleep (Hobson et al., 2000). According to Hobson and McCarley's (1977) activation-synthesis model, the brainstem contains a dream-state generator that periodically activates a great number of subcortical and cortical structures during the REM stage. The brainsteminitiated neural activation is most pronounced in the visual cortex, the motor cortex, the basal ganglia, and various limbic system structures, particularly the amygdala (Braun et al., 1997; Maquet et al., 1996; Nofzinger, Mintun, Wiseman, Kupfer, \& Moore, 1997). The pattern of activation during REM corresponds closely with dream content and matches the neural activity of the same behaviors during waking consciousness (see Hobson et al., 2000).

PET studies have also revealed significant deactivation of a vast area of the DL, including areas 8, 9, 10, 11, 46, 47 (Braun et al., 1997; Maquet et al., 1996). The pattern of activation is so striking that "REM sleep may constitute a state of generalized brain activity with the specific exclusion of the executive system" (Braun et al., 1997, p. 1190). Phenomenologically, dream stories are void of prefrontal-dependent cognition. Self-reflection is absent (Rechtschaffen, 1978), time is distorted with past, present, and future freely exchanged (Hobson, 1988), and volitional control is greatly diminished (Hartman, 1966). There is also little abstract, 'bigger picture' thinking, little active decision making, little consistent logic, a crude selfconstruct, and not much indication of theory of mind capabilities or focused attention. The capacity for semantic and episodic retrieval of specific memories, which heavily relies on DL areas (see Cabeza \& Nyberg, 2000), is also greatly decreased. Furthermore, the extent to which a dream is bizarre is related to the extent of the prefrontal hypofunction (Hobson et al., 2000). It is evident that the principal difference between dream mentation and normal waking consciousness is due to the deactivation of the DL. 
For the study of consciousness, lucid dreaming is an imperative subjective alteration of the dream state. During a lucid dream, a person becomes aware that the transpiring events are part of a dream (LaBerge, 1985). Hobson (2001) has defined lucid dreaming "as the bolstering of the self-reflective awareness that is normally diminished or absent in dreaming" (p. 93). The degree of lucidity is variable and it is not necessarily accompanied by dream control, the intentional directing of the dream story. Similarly dream control can occur without lucidity (LaBerge, 1985). Hobson (2001) has proposed that lucid dreaming is the result of DL activation during REM sleep, or more specifically "the residual activation of the dorsolateral prefrontal cortex [that] is amplified by the REM activation" (p. 97) from other cortical areas. Psychological reports from lucid dreamers are indicative of other prefrontal cognitive capacities that are normally absent in dreaming, such as willed action, an undistorted sense of self, directed attention, abstract thinking in the form of constructive creativity, consciously operated memory searches, awareness of complex societal standards, and strategic planning. LaBerge (1985) has also shown in his laboratory that the estimation of the passage of time during a lucid dream is as accurate as in waking consciousness. Additionally, the degree and quality of the lucid experience might be a function of the extent and pattern of prefrontal activation. The observation that dream control and lucidity, although highly correlated, are independent might underscore this notion. With the top layers of consciousness activated, lucid dreaming is more akin to waking consciousness, neurally as well as psychologically. Indeed, according to LaBerge (1985), awakening is one of the most common results of suddenly gaining lucidity in a dream. In that sense, lucid dreaming might be better thought of as daydreaming during REM sleep.

\subsection{Endurance running}

An exercise induced altered state of consciousness has long been appreciated by endurance athletes. The effect has been well documented and subjected to scientific investigation (e.g., Hoffman, 1997; Mandell, 1981; Pargman \& Baker, 1980), and since the effect is particularly prominent in long-distance running, the phenomenon was given its contemporary descriptor, the "runner's high" (Pargman \& Baker, 1980). Phenomenologically, the runner's high has been described as pure happiness, elation, feelings of unity with one's self and/or nature, endless peacefulness, timelessness, inner harmony, boundless energy, as well as the reduction of pain sensations (e.g., Farrell, Gustafson, Morgan, \& Pert, 1987; Hoffman, 1997). These subjective descriptions are similar to the claims of distorted perception, atypical thought patterns, diminished awareness of one's surroundings, and intensified introspective understanding of one's sense of identity and emotional status made by people who describe trance states. Moreover, exercise has well-established beneficial effects on mood states, particularly stress, anxiety, and depression, (for reviews, see Glenister, 1996; Salmon, 2001; Scully, Kremer, Meade, Graham, \& Dudgeon, 1998).

There is extensive literature documenting depression as a result of frontal lobe damage (for a review, see Starkstein \& Robinson, 1999). PET neuroimaging studies have also demonstrated significant hypometabolism in the DL of patients with depression (Baxter et al., 1987). This may explain a depressed patient's persever- 
ance on negative thoughts and the inability to shift away from negative events. Without the cognitive flexibility of a fully functional DL, patients might find themselves unable to escape from negative environmental cues. PET studies have further demonstrated that the VM, along with the amygdala and the anterior cingulate, is hyperactive during depression (see Mayberg, 1997). This hypermetabolism may explain why depressed patients overemphasize the importance of negative events in their lives, especially with sustained and heightened amygdala input. Healthy subjects that are asked to think sad thoughts show similar hypermetabolism in the VM, while metabolic activity is less than normal for DL circuits (Damasio et al., 2000). These studies point to an abnormal interaction between the VM and the DL rather than global prefrontal dysfunction (Starkstein \& Robinson, 1999). PET studies of anxiety disorders show a very similar prefrontal pattern (Ruben \& Harris, 1999). In obsessive-compulsive disorder (OCD) for instance, the VM also exhibits widespread hypermetabolism but without the concomitant limbic hypermetabolism (Baxter, 1990).

Based on the prefrontal pathology in depression and anxiety disorders, I have proposed the hypothesis that exercise induces a state of transient hypofrontality, which could account for the beneficial effects of exercise on mental health (Dietrich, in review). A brief reiteration of the main points and the line of reasoning might be beneficial to the continuity of this article.

Neuroimaging techniques demonstrate that different tasks place different demands on neural structures, which results in unique patterns of activation. Considering the physical demands of exercise, it is reasonable to assume that, at a minimum, there is activation in all neural structures that are required in order to run the motor patterns to sustain the physical activity: primary motor cortex, secondary motor cortices, basal ganglia, cerebellum, various midbrain nuclei (red and substantia nigra), as well as various thalamic nuclei would be involved. Depending on the type of sport, exercise should result in activity of structures involved in perceptual function and memory, particularly TOP and the sensory thalamus. Local cerebral glucose metabolism and utilization measured in running rats has confirmed this pattern of activity (Vissing, Anderson, \& Diemer, 1996). Since such a large part of the brain is devoted to perception and motor output, there is a widespread activation of numerous structures across the entire brain during exercise. Yet, it is important to clarify at this point that, despite regional increases, global cerebral blood flow to the brain during exercise as well as global oxygen uptake is constant (Ide \& Secher, 2000).

Research in cognitive psychology has shown that humans have a limited information processing capacity, particularly with respect to attentional resources (Broadbent, 1958; Cerry, 1953). This is likely due to the brain's limited resources and the inability to sustained activation in all neural structures at once. As Pinker (1999) has pointed out, there are costs and benefits associated with efficient information processing. It was suggested that the widespread activation of motor and sensory systems during exercise comes at the expense of, first and foremost, the higher cognitive centers of the prefrontal cortex. Also, limbic system structures such as the amygdala that are not directly required for the exercise might similarly show depressed activity. 
EEG recordings of exercising individuals seem to be consistent with this hypothesis. Exercise is associated with $\alpha$-enhancement, particularly in the frontal cortex (e.g., Kubitz \& Pothakos, 1997). The increase in $\alpha$-activity during and after exercise has been interpreted in the literature as indicative of decreased brain activation (Kubitz \& Pothakos, 1997). Single cell recording in exercising cats has also provided support for decreased activation in prefrontal regions. Recording from 63 neurons in the prefrontal cortex, units associated with the control of the movement showed increased activity during locomotion, while other prefrontal units decreased their discharge (Criado, de la Fuente, Heredia, Riolobos, \& Yajeya, 1997).

It is currently impossible to test the hypothesis directly with functional imaging tools because they preclude head movement. However, specific predictions can be made using cognitive tests that are indicative of a specific brain region's viability. For instance, according to the hypothesis, an individual's ability to perform frontal-dependent cognitive tasks during exercise should be impaired. It was noted early (e.g., Hebb, 1939) that people with frontal lobe lesions perform normally on conventional intelligence tests, suggesting that successful performance on IQ tests does not require a fully functional frontal lobe. The transient hypofrontality hypothesis predicts that endurance running might produce greater deficits in frontal lobe sensitive tasks than with conventional IQ tests. This experiment was recently performed in our laboratory and it has confirmed that endurance exercise selectively impairs frontal-dependent cognition (Dietrich \& Sparling, in review). In addition, other possible tests of the transient hypofrontality hypothesis might make use of tools such as optical imaging, single cell recording in animals, EEG recordings in humans, as well as a variety of different cognitive and emotional test batteries for humans and animals that test for psychological processes that are prefrontal-dependent.

A state of transient frontal hypofunction can explain a variety of emotional and cognitive changes that are experienced during exercise. On a psychological level, it has been suggested that exercise's mental health benefits might simply be due to distraction or a 'time-out' from life's stress (Bahrke \& Morgan, 1978). Exercise-induced transient hypofrontality could represent a neural origin for this distraction. Given the analytical and attentional capacities of the prefrontal cortex, excessive activity generates a state of hyper-vigilance and hyper-awareness. In such a state, there is a tendency to overanalyze and evaluate every event with respect to personal relevance. Exercise might simply 'take the edge off' by neutralizing this circuitry, producing an inability to focus on life's worries.

Although the hypothesis of exercise-induced transient hypofrontality was developed to account for the beneficial effects of exercise on mental health, the prolonged disengagement of higher cognitive centers in the prefrontal cortex also offers a neural mechanism that provides insight into the alteration of consciousness known as the runner's high. Some of the phenomenologically unique features of this state such as experiences of timelessness, living in the here and now, reduced awareness of one's surroundings, peacefulness (being less analytical), and floating (diminished working memory and attentional capacities), are consistent with a state of frontal hypofunction. Even abstruse feelings such as the unity with the self and/or nature might be more explicable, considering that the prefrontal cortex is the very structure that provides us with the ability to segregate, differentiate, and analyze the environment. 
The hypothesis proposes further that the degree to which the prefrontal cortex is free to disengage from an on-going activity is dependent upon the demands made by the physical activity on these higher cognitive functions. Thus, clear predictions can be made about how different types of sports affect brain function. Strategic team sports such as basketball require a variety of frontal-dependent cognitive processes while running through a familiar park does not. The hypothesis also predicts that intensity is important. A minimum level of intensity is required to force the redistribution of resources in the brain. Conversely, exercise that is extremely strenuous and associated with high levels of discomfort and fatigue lead to ubiquitous awareness and alarm signals, that forces prefrontal regions to evaluate the person's status and situation.

The transient hypofrontality hypothesis also predicts that the experience of an "exercise high" depends on the skill level and the nature of the movement. It has been suggested that automatic motor behaviors, or habits, are controlled by the basal ganglia (Mishkin, Malamut, \& Bachevalier, 1984). The more a skill is practiced and becomes automatic, the less prefrontal cortex activity is required during its execution. Hence, skill level, or the degree to which a motor pattern is run by the basal ganglia, has an affect on how much an individual can afford to deregulate the prefrontal cortex. Similarly, the more natural the movement, the more rapidly the movement is transferred to the basal ganglia. Thus, the natural activity of running would more readily produce a 'high' than perhaps swimming or yoga.

\subsection{Meditation}

Meditation has long been regarded as an altered state of consciousness. Although there are many different forms and practices of it, all share a number of common features. First, sensory input is gradually diminished in the meditative state, creating what Carrington (1998) called "a mental isolation chamber" (p. 68). The continuous sensory bombardment of daily life is ultimately replaced by a non-judgmental, receptive, quiescent state that deliberately banishes the intrusion of new input. This feature by itself might explain many well-documented meditation effects such as increased relaxation, reduced anxiety, lowered blood pressure, or changes in cortisol levels (for a review, see Carrington, 1998; Jevning, Wallace, \& Beidebach, 1992).

Second, meditation entails sustained concentration and heightened awareness by focusing attention on a mantra, breathing rhythm, or a number of other internal or external events. This increase in attentional effort serves to accomplish the exclusion of other, intruding information. The attention is directed, deliberate, and sustained, suggesting the activation of the frontal attentional network. Indeed, all existing studies using neuroimaging techniques during meditation show converging evidence of DL activation (Herzog et al., 1990; Lazar et al., 2000; Lou et al., 1999; Newberg et al., 2001). At first glance, these data appear to contradict the transient hypofrontality hypothesis of altered states of consciousness.

Paradoxically, early EEG studies of meditation consistently detected $\alpha$-wave activity across the frontal lobe (Anand, China, \& Singh, 1961; Banquent, 1972; Benson, Mahlotra, Goldman, Jacobs, \& Hopkins, 1990; Corby, Roth, Zarcone, \& Kopell, 1978). $\alpha$-Activity consists of regular, smooth medium-frequency waves, 
which are produced when subjects are neither particularly aroused nor excited, and are not engaged in strenuous mental activity such as problem solving. In short, $\alpha-$ activity suggests decreased brain activation. Conversely, $\beta$-activity is an irregular wave pattern that is detected when subjects are alert and attentive. Given that meditation increases attentional focus and awareness, as suggested by the psychological reports and neuroimaging data, one would expect to detect $\beta$-activity across the frontal lobe during meditation. Or in more esoteric terms, if meditation is, as so often claimed, a vehicle for reaching 'higher consciousness' one would expect the neural structure that enables the highest cognitive functions and thus the top layers of consciousness to be fully functional. The presence of $\alpha$-waves in EEG studies are in stark contrast to the neuroimaging data showing increased neural activity in the prefrontal cortex.

EEG is a crude method that measures summed postsynaptic electrical activity of a large area of the cortex. The desynchronized pattern of $\beta$-activity is due to the differential activation of individual but spatially overlapping circuits or modules, resulting in a noisy, overall signal. The neural synchrony that is reflected by $\alpha$-activity indicates that large groups of neurons fire in unison. This has been, often presumptuously, interpreted as the neural correlate of psychological harmony when it is perhaps more indicative of baseline cortical activity or metabolic depression.

It is evident that more research is needed to resolve the conflicting EEG and neuroimaging data. Reinterpreting and integrating the limited data from existing studies, it is proposed that meditation results in transient hypofrontality with the notable exception of the attentional network in the prefrontal cortex. The resulting conscious state is one of full alertness and a heightened sense of awareness, but without content. Since attention appears to be a rather global prefrontal function (e.g., Cabeza \& Nyberg, 2000), PET, SPECT, and fMRI scans showed an overall increase in DL activity during the practice of meditation. However, the attentional network is likely to overlap spatially with modules subserving other prefrontal functions and an increase as measured by fMRI does not inevitably signify the activation of all of the region's modules. Humans appear to have a great deal of control over what they attend to (Atkinson \& Shiffrin, 1968), and in meditation, attentional resources are used to actively amplify a particular event such as a mantra until it becomes the exclusive content in the working memory buffer. This intentional, concentrated effort selectively disengages all other cognitive capacities of the prefrontal cortex, accounting for the $\alpha$-activity. Phenomenologically, meditators report a state that is consistent with decreased frontal function such as a sense of timelessness, denial of self, little if any self-reflection and analysis, little emotional content, little abstract thinking, no planning, and a sensation of unity. The highly focused attention is the most distinguishing feature of the meditative state, while other altered states of consciousness tend to be more characterized by aimless drifting.

It is well documented that meditation also reduces anxiety and alleviates mild depression (Carrington, 1998). As mentioned earlier, depression and anxiety are believed to be due, in part, to a differential activation of VM and DL circuits (Starkstein \& Robinson, 1999). Treatment with selective serotonin reuptake blockers normalizes this imbalance (Mayberg, 1997). Although highly speculative at this 
point, it is proposed that any selective boosting of DL activity, such as in meditation, should have an antidepressant and anxiolytic effect. In a similar manner, talking to a friend or therapist about an emotional dilemma involves sustained attention, abstract thinking, and formulating explanations so the person can understand the situation. This should also require DL activation and thus the reactivation of circuits that can modulate the perseverance on negative thinking. Normalizing DL activity should result in increased cognitive flexibility and thus enhance the ability to extract oneself from the perceived dilemma.

\subsection{Hypnosis}

Although still viewed with skepticism, hypnosis has gained respectability in medicine, in large part, due to its demonstrated effects on analgesia. It is less clear however whether hypnosis constitutes an altered state of consciousness. Forty percent of hypnotized subjects describe it as an altered state, while sixty percent compare it to a state of focused attention (Kirsch \& Lynn, 1998). While some researchers postulated a divided stream of consciousness in hypnosis (Hilgard, 1980), others caution that the evidence only supports a state of high suggestibility (e.g., Barber, 1970; Kirsch \& Lynn, 1998; Spanos, 1994). Hypnotized subjects reported or exhibited analgesia, vivid images, hallucinations in all sense modalities, amnesia, timelessness, detachment from the self, and a willingness to accept distortions of logic and reality (e.g., Kihlstrom, 1985; Orne, 1959; Tart, 1979). More commonly, hypnotized individuals remain fully alert, conversing with people and navigating the environment normally. The main distinguishing characteristic of the hypnotic state remains the lack of initiative or willful movement.

Early EEG studies failed to uncover an unambiguous physiological marker of hypnosis (Benson, Arns, \& Hoffman, 1981). Conversely, recent neuroimaging studies have demonstrated subtle changes in neural activation, particularly in the left hemisphere (Maquet et al., 1999; Rainville et al., 1999; Wik, Fischer, Bragee, Finer, \& Frederikson, 1999). The observed activation occurred predominantly in TOP cortices without a corresponding increase in DL circuits. Increased activation was also observed in the ventrolateral prefrontal cortex during hypnotic suggestion, presumably reflecting the demand on attentional resources in order to process the verbal input (Rainville et al., 1999; Wik et al., 1999). This was also shown in an earlier regional blood flow study (Crawford, Gur, Skolnick, Gur, \& Benson, 1993). This pattern of activation was not found in neuroimaging studies using other dependent and independent variables of hypnosis (Faymonville et al., 2000; Ground, Pawlik, Walter, Lesch, \& Heiss, 1995).

A number of researchers have postulated that neural structures compete for access to consciousness (e.g., Baddeley, 1995; Pinker, 1999). The process of hypnotic induction, regardless of how it is implemented, serves to narrow a person's attention. It has been suggested that the effects of hypnosis are due to frontal inhibition (for recent reviews see, Gruzelier, 2000; Kallio, Revonsuo, Hämäläinen, Markela, \& Gruzelier, 2001). The transient hypofrontality hypothesis suggests further that, similar to meditation, the focused attention of the hypnotic state is the mechanism by which the activation of various prefrontal circuits is decreased, eliminating their 
computation from figuring into immediate conscious experience. It appears that some cognitive functions supported by the DL prefrontal cortex such as willed action, initiative, critical self-reflection, memory accessibility, cognitive flexibility, and independent thinking and logic are particularly affected in hypnosis.

It is proposed that during hypnosis, the hypnotist's suggestions, due to the subject's narrow focus on them, become the predominant content in the working memory buffers without passing through the filter of these other downregulated DL circuits. Without the added higher cognitive computation provided by these DL circuits, the person does not have the capacity to critically examine the suggestions and thus enable behavioral flexibility. It is evident that the hypofunction does not appear to be a total or global prefrontal event. For instance, it has been demonstrated that hypnotized subjects cannot be induced to act contrary to their moral beliefs and values (Kirsch \& Lynn, 1998), which is primarily a function of the VM (Damasio, 1994). This is supported by neuroimaging studies showing VM activation (Maquet et al., 1999; Rainville et al., 1999). It is also suggested that the experience of a hidden observer and a divided stream of consciousness postulated by Hilgard (1980) might be due to prefrontal circuits that are not altered by the hypnotic induction. Similar to lucid dreaming, these 'indomitable' circuits may provide lucidity without being able to influence real actions in real life. In hypnosis, suggestions become executed by directly activating the motor system without being further scrutinized. This is in accordance with people's subjective description of the hypnotic experience, stating that a given behavioral act appeared to happened by itself.

As mentioned earlier, Lhermitte (1983) and Lhermitte et al. (1986) documented a strong tendency for frontal lobe patients to depend on immediate environmental cues to guide their behavior. They appear to be compelled to use objects they see and to imitate the behavior they observe in others. Such utilization behavior is astoundingly reminiscent of hypnosis; only with frontal lobe patients the suggestions are environmental triggers and not verbal commands. As with hypnosis, the susceptibility to suggestions in frontal lobe patients is accompanied by a concomitant lack of initiative, perseverance of thought, lack of independent thinking, and decreased behavioral flexibility. It is also interesting to note that children tend to be more hypnotizable (Plotnick, Payne, \& O'Grady, 1991). The prefrontal cortex is still in the developmental stage and children show imitation behaviors with a much higher frequency. The willingness to accept apparent contradictions in logic during hypnosis is also reminiscent of the logic used by children. Children are unable, until a certain age, to reflect on and integrate logical inconsistencies and do not appear to be bothered by their existence. Research shows that young adults with frontal brain damage perform at the level of six- or seven-year-olds on Piaget's inclusion or other categorization tasks (Houde \& Joyes, 1995), demonstrating the intimate link between logical reasoning ability and prefrontal cortex viability. In hypnosis, logic is similarly suspended (Orne, 1959), further implicating prefrontal circuits.

The peculiar properties of hypnotic analgesia further point to the involvement of the prefrontal cortex in hypnosis. For instance, a patient might experience pain-free surgery while still complaining about the cold temperature in the operating room (Hardcastle, 1999). Similarly, hypnotized subjects might report pain in one hand but not in the another, although both are subjected to the same treatment. Hardcastle 
(1999) pointed out that, to intentionally ignore some specific pain, one would have to notice it first in order to know what pain to ignore. In other words, the sensation must be recognized, distinguished from other sensations, and then selectively blocked from figuring into conscious awareness. This suggests a top down process of the highest order. Frontal lobe patients show a strikingly similar analgesic phenomenon in that they report the experience of pain but show little, if any, signs of being distressed by it (Vertosick, 2000). It has been suggested that the increase in blood flow over the VM prefrontal cortex might reflect the attention system's effort to keep the emotional salience of the sensation from reaching consciousness (Crawford et al., 1993).

These observations are consistent with the frontal hypothesis of hypnosis (e.g., Gruzelier, 2000). Direct evidence for this notion has come from testing hypnotized subjects using various neuropsychological measures such as the Stroop test that depend on prefrontal activation. Results from these studies showed that subjects perform poorly under hypnosis as compared to baseline (Dixon \& Laurence, 1992; Nordby, Hugdahl, Jasiukaitis, \& Spiegel, 1999; Kaiser, Barker, Haenschel, Baldeweg, \& Gruzelier, 1997; Kallio et al., 2001; Sheehan, Donovan, \& MacLeod, 1988). In addition, studies measuring event-related potentials during hypnosis also indicate decreased prefrontal activation (Nordby et al., 1999; Kaiser et al., 1997). Further research using neuropsychological tests that are more indicative for various prefrontal functions such as working memory (e.g., WCST or a delayed non-matching to sample task), sustained attention (e.g., PASAT), or specific higher cognitive functions such as theory of mind paradigms, could help identify in more detail specific prefrontal circuits that are affected by the hypnotic induction.

\subsection{Daydreaming}

In meditation and hypnosis, subjective experience is intentionally altered through the ability to control and direct attentional resources. Since these resources are limited, neural activity not brought into focus is excluded from consciousness. In contrast, daydreaming is (1) not necessarily intentional and (2) not a redirection of attentional focus, but simply a loss of attentional power. Consequently, daydreaming is not a state of high alertness, but characterized by drifting, ephemeral thoughts and a sense of timelessness. However, all three alterations of consciousness share the feature of toning-down external noise as a prerequisite to enable the mental state.

Humans daydream frequently (Gold, Gold, \& Milner, 1987; Ray \& Faith, 1995). Given the astronomical amount of sensory information, attention is believed to be "the result of a limited information processing capacity" (Broadbent, 1958, p. 68). Research in cognitive psychology has shown that focused attention is a taxing mechanism and cannot be maintained indefinitely. The shifting of attentional style towards internal events during daydreams (Singer, 1978) might simply be the inevitable result of the constant demands placed on the attentional system to selectively processing novel information. Daydreaming goes dramatically down with age, suggesting that it might be necessary for normal, healthy brain development (Singer, 
1975). Given the limited attentional capacity, the brain must downregulate external awareness in order to assimilate the massive amount of input. Either way, daydreaming is an integral part of conscious life (Singer \& Pope, 1981).

While daydreaming, humans are capable of an astonishingly high level of processing. A common example is the experience of daydreaming while driving a car on a familiar route. As consciousness is engaged in a daydream scenario, the driver navigates complex traffic patterns without paying conscious attention to them. If daydreaming is a loss of attentional power and a toning down of external stimuli, how can the driver steer through ever-changing traffic situations?

Attention occurs at all levels of information processing (Taylor, 2001) but directed, sustained attention is a prefrontal function (e.g., Posner, 1994). As mentioned earlier, research has shown that attention at the prefrontal level is a serial process, and humans are capable of two behaviors simultaneously only if one is automatic (Broadbent, 1958; Cerry, 1953). Given that automatic motor behaviors are controlled by the basal ganglia (Mishkin et al., 1984), the more a skill is practiced and becomes automatic, the less prefrontal cortex activity is required during its execution. Hence, while performing a routine task, the prefrontal cortex is permitted to generate a daydream scenario. At any time, the control can be transferred back to the prefrontal cortex, for instance, if an unusual event occurs during the drive home. These and other data strongly suggest that consciousness is a singular process, while the unconscious brain appears to be a parallel processor (Gazzaniga, Ivry, \& Mangun, 1998). The unity of consciousness might be a result of limited attentional capacity (Posner, 1994).

Given the notions that working memory holds in mind the current content of consciousness, and that attention is the mechanism to select the content, basal ganglia controlled motor output bypasses consciousness. As mentioned earlier, there is selectivity of sensory information at all levels of perceptual processing. The parietal cortex and a number of higher visual cortices such as the inferior temporal cortex have been implicated in intermediate level or "central" attentional processes (Taylor, 2001). As suggested by frontal lobe patients, these processes are sufficient to navigate one's changing environment. Parietal and temporal cortical areas have direct connections to the basal ganglia and it has been suggested that such input guides basal ganglia motor output (Ashby, Isen, \& Turken, 1999), while the working memory buffer contains the daydream scenario. In a similar manner, highly sophisticated cognitive processes may occur in other altered states of consciousness such as sleepwalking or hypnosis.

\subsection{Drug-induced altered states}

Drugs have been used throughout history to alter consciousness. Due to the known mechanisms of action of many drugs and their widespread use, theories of altered states remain focused on neurotransmitter modulation (e.g., Hobson, 2001). Neurochemical explanations of brain function and brain disorders have been equally popular. From monoamine and $\beta$-adrenergic theories of depression to the dopamine theory of schizophrenia, neurotransmitters have been credited with functions as diverse as mood, memory, attention, or consciousness. 
There is a danger of leaning too hard on this concept. Each neurotransmitter system can be modulated by drugs that do or do not alter consciousness. It is important to point out that neurotransmitters and neuromodulators do not carry content in their messages. While chemical neurotransmission and synaptic changes certainly play an important role in regulating brain function and thus consciousness, it is similarly important to understand the function of the neural structure in which the synaptic change occurs.

Neural structures were molded by evolutionary pressures. Neuronal cells in a given structure migrated and differentiated according to a genetic code, forming circuits that execute a specific neural computation. A change in the neural structure will alter the way the mind operates in a particular way, regardless of the neurotransmitter the structure evolved to utilize. In other words, brain chemicals work globally and the same neurotransmitter/receptor coupling or the same synaptic modulation in one structure will produce a different effect in another structure. Thus, it is crucial to understand the fundamental task performed by the relevant neural structure.

Drugs that work on the same neurotransmitter system, even via the same synaptic mechanism within that system, can have different behavioral effects (Diaz, 1997). For instance, cocaine and Ritalin are both dopamine reuptake blockers, but while one drug induces sleepless nights, the other is used to calm children with attention deficit disorder. Most transmitter systems have multiple receptor types and subtypes. The density and number of these receptor populations vary widely from structure to structure, resulting in differential binding profiles for different drugs and thus different functional changes. It is not the molecule per se that determines drug effects, but also the location of its receptors and thus the neural structure whose function is altered.

Hence, theories of drug-induced altered consciousness solely based on neurochemical changes are inadequate. A comprehensive theory of these states must consider functional neuroanatomy. The transient hypofrontality hypothesis proposes that psychoactive drugs that induce an altered state of consciousness do so by temporarily decreasing prefrontal viability, either as a direct consequence of the drug's primary action or as a secondary consequence. Each drug causes a unique phenomenological awareness that can be discriminated from one caused by another drug (Siegel, 1985). Yet all drug-induced altered states, regardless of the drug that generated them, share a number of prominent characteristics that are consistent with a state frontal inhibition. To various degrees, all generate sensations of timelessness, decrease attentional and working memory ability, produce a lack of strategic planning, enhance perseverance, and increase social impropriety (e.g., Diaz, 1997). Additionally, numerous drugs, particularly the psychedelics, cause distortions of the self, dream-like detachment from reality, and a lack of cognitive flexibility evidenced by the inability to extract oneself from the here and now of the experience (Diaz, 1997; Siegel, 1985). In that sense, it is suggested that drugs are not mind-expanding but rather mind-reducing, as they limit the maximum capacity for consciousness.

The fact that these similarities in cognitive impairment are induced by drugs that belong to different classes and have different mechanisms of action suggests that all inhibit prefrontal activity in some way. Of course, this is not to say that all 
psychoactive drugs deregulate prefrontal activity. For instance, psychoactive drugs such as Prozac or Ritalin do not alter prefrontal-dependent higher cognitive functions (Diaz, 1997). However, these drugs also do not induce an altered state of consciousness. Unlike non-chemical induced altered states, drugs may also cause excessive neural activity. Depending on the affected region, this should result in phenomenological addition. Thus, the unique phenomenological state that is induced by a particular drug might be the result of the drug's primary effect on a particular neurotransmitter system as well as the downregulation of a particular set of prefrontal circuits. For instance, fMRI studies of marijuana users show excessive activity in the medial forebrain bundle and other mesolimbic circuits, while showing a decrease in neural activity in the DL (Loeber \& Yurgelun-Todd, 1999; Pistis, Porcu, Melis, Diana, \& Gessa, 2001). Cocaine and ecstasy show similar neural profiles (Bolla, Cadet, \& London, 1998; Hegadoren, Baker, \& Bourin, 1999; Kalant, 2001). Whatever information is 'online' at the time is used by the residual capacity of the prefrontal cortex to enable an ontological reality. Consequently, it is suggested that the greater the differential between prefrontal and other brain activity, the more bizarre the experience. Given the distribution of individual neurotransmitter systems in the brain and the specificity of certain drugs to particular receptors, it is evident that some chemical substances produce a greater disparity and thus a more profound alteration to consciousness.

\section{Concluding remarks}

Relevant data and supportive evidence from psychology and neuroscience were reviewed in order to establish a theoretical basis for the transient hypofrontality hypothesis of altered states of consciousness. Although different behavioral methods are used to achieve different states, it is proposed that all altered states share a common neural mechanism; that is a transient decrease in prefrontal cortex activity. Furthermore, it is hypothesized that different induction methods target specific prefrontal circuits, removing their computation from the conscious experience. This distinct phenomenological subtraction accounts for the uniqueness of each altered state.

For each altered state, a behavioral technique induces neural changes that compromise the viability of the prefrontal cortex, and thus produce subtle changes in the content of consciousness. In meditation, hypnosis, and daydreaming, we make use of our ability to control attentional resources in order to eliminate extraneous information from being processed consciously. This intentional blockage permits specific prefrontal circuits to be run in 'safe mode.' While in meditation and hypnosis attention is redirected, daydreaming accomplishes this feat by reducing attentional ability. Likewise, REM sleep is a state of reduced environmental awareness with the concomitant inactivity in prefrontal regions, except that in this altered state the event is much more profound and induced involuntarily by a circadian rhythm. In longdistance running, the demands of the physical workload force the redistribution of neural resources in the brain. Given our limited information processing capacity, it is suggested that sports that do not require frontal-dependent cognition will disengage 
the prefrontal cortex from on-going activities, making those sports more conducive to alter consciousness. Finally, evidence strongly suggests that the administration of drugs deactivates prefrontal regions.

The transient hypofrontality hypothesis attempts to unify the disparate literature on altered states of consciousness by proposing a common neural mechanism that can be subjected to vigorous assessment in the laboratory. The hypothesis is based on considerations of the functional neuroanatomy of the prefrontal cortex and offers a feasible alternative to neurochemical explanations. Unlike other theories, a state of diminished metabolism in prefrontal regions can account for a wide variety of well-documented psychological and neurological effects of altered states. It is hoped that the heuristic value of the hypothesis will stimulate research and will encourage researchers from diverse backgrounds to address testable hypotheses derived from it.

\section{Acknowledgments}

I thank Thomas Burkholder, Bill McDaniel, Phil Sparling, and Tony Johnson for valuable suggestions and comments. I also thank Charles Selye for help with the figure.

\section{References}

Anand, B. K., China, G., \& Singh, B. (1961). Some aspects of electroencephalographic studies in yogies. Electroencephalogry and Clinical Neurophysiology, 13, 452-456.

Ashby, G. F., Isen, A. M., \& Turken, A. U. (1999). A neuropsychological theory of positive affect and its influence on cognition. Psychological Review, 106, 529-550.

Atkinson, R. C., \& Shiffrin, R. M. (1968). Human memory: A proposed system and its control processes. In K. W. Spence \& J. T. Spence (Eds.), The psychology of learning and motivation: Advances in research and theory (Vol. 2, pp. 89-195). New York: Academic Press.

Baars, B. J. (1989). A cognitive theory of consciousness. Cambridge: Cambridge University Press.

Baars, B. J. (1995). Tutorial commentary: Surprisingly small subcortical structures are needed for the state of waking consciousness, while cortical projection areas seem to provide perceptual content of consciousness. Consciousness and Cognition, 4, 159-162.

Baddeley, A. (1995). Working memory. In M. S. Gazzaniga (Ed.), The cognitive neurosciences (pp. 755-764). Cambridge, MA: MIT Press.

Baddeley, A. (2000). The episodic buffer: A new component of working memory. Trends in Cognitive Sciences, 4, 417-423.

Bahrke, M. S., \& Morgan, W. P. (1978). Anxiety reduction following exercise and meditation. Cognitive Therapy and Research, 2, 323-334.

Banquent, J. P. (1972). EEG and meditation. Electroencephalogry and Clinical Neurophysiology, 33, 454.

Barber, T. X. (1970). Who believes in hypnosis? Psychology Today, 4, 20-27.

Baxter, L. R. (1990). Brain imaging as a tool in establishing a theory of brain pathology in obsessive-compulsive disorder. Journal of Clinical Psychiatry, 51(Suppl.), 22-25. 
Baxter, L. R., Phelps, M. E., Mazziotta, J. C., Guze, B. H., Schwartz, J. M., \& Selin, C. E. (1987). Local cerebral glucose metabolic rates in obsessive-compulsive disorder: A comparison with rates in unipolar depression and normal controls. Archives of General Psychiatry, 44, 211-218.

Benson, H., Arns, P. A., \& Hoffman, J. W. (1981). The relaxation response and hypnosis. International Journal of Clinical and Experimental Hypnosis, 29, 259-270.

Benson, H., Mahlotra, M. S., Goldman, R. F., Jacobs, G. D., \& Hopkins, P. J. (1990). Three case reports of the metabolic and electroencephalographic changes during advanced Buddhist meditation techniques. Behavioral Medicine, 16, 90-95.

Bogen, J. E. (1995). On the neurophysiology of consciousness. I. An overview. Consciousness and Cognition, 4, 137-158.

Bolla, K. I., Cadet, J. L., \& London, E. D. (1998). The neuropsychiatry of cocaine abuse. Journal of Neuropsychiatry and Clinical Neuroscience, 10, 280-289.

Brauer Boone, K. (1999). Neuropsychological assessment of executive functions. In B. L. Miller \& J. L. Cummings (Eds.), The human frontal lobes: Functions and disorders (pp. 247260). New York: Guilford Press.

Braun, A. R., Balkin, T. J., Wesensten, N. J., Gwadry, F., Carson, R. E., Varga, M., Baldwin, P., Selbie, S., Belenky, G., \& Herscovitch, P. (1997). Regional cerebral blood flow throughout the sleep-wake cycle. Brain, 120, 1173-1197.

Brickner, R. M. (1936). The intellectual function of the frontal lobe: Studies based upon observation of a man after partial bilateral frontal lobotomy. New York: Macmillan.

Broadbent, D. A. (1958). Perception and communication. New York: Pergamon.

Cabeza, R., \& Nyberg, L. (2000). Imaging cognition II: An empirical review of 275 PET and fMRI studies. Journal of Cognitive Neuroscience, 12, 1-47.

Carrington, P. (1998). The book of meditation: The complete guide to modern meditation (revised ed.). New York: Element Books.

Cerry, E. C. (1953). Some experiments on the recognition of speech, with one and two ears. Journal of Acoustic Society American, 25, 975-979.

Corby, J. C., Roth, W. T., Zarcone, V. P., \& Kopell, B. S. (1978). Psychophysiological correlates of the practice of Tantric Yoga meditation. Archives of General Psychiatry, 35, 571-577.

Courtney, S. M., Petit, L., Haxby, J. V., \& Ungerleider, L. G. (1998). The role of prefrontal cortex in working memory: Examining on the contents of consciousness. Philosophical Transactions of the Royal Society of London Series B: Biological Sciences, 353, 18191828.

Crawford, H. J., Gur, R. C., Skolnick, B., Gur, R. E., \& Benson, D. M. (1993). Effects of hypnosis on regional blood flow during ischemic pain with and without suggested hypnotic analgesia. International Journal of Psychophysiology, 15, 181-195.

Criado, J. M., de la Fuente, A., Heredia, M., Riolobos, A. S., \& Yajeya, J. (1997). Electrophysiological study of prefrontal neurons of cats during a motor task. European Journal of Physiology, 434, 91-96.

Crick, F. H. C. (1994). The astonishing hypothesis. New York: Scribner.

Crick, F. H. C., \& Koch, C. (1998). Consciousness and neuroscience. Cerebral Cortex, 8, $97-$ 107.

Damasio, A. R. (1994). Descartes' error: Emotion, reason, and the human brain. New York: G.P. Putnam.

Damasio, A. R. (1999). The feeling of what happens. New York: Hartcourt Brace.

Damasio, A. R., Graboweski, T. J., Bechera, A., Damasio, H., Ponto, L. L. B., Parvizi, J., \& Hichwa, R. D. (2000). Subcortical and cortical brain activity during the feeling of selfgenerated emotions. Nature Neuroscience, 3, 1049-1056. 
Dehaene, S., \& Naccache, L. (2001). Towards a cognitive science of consciousness: Basic evidence and a workspace framework. Cognition, 79, 1-37.

Diaz, J. (1997). How drugs influence behavior: A neurobehavioral approach. Upper Saddle River, NJ: Prentice-Hall.

Dietrich, A. (in review). Exercise, mental health and neuroscience: A new hypothesis. Psychological Science.

Dietrich, A., \& Sparling, P. B. (in review). Endurance exercise selectively impairs prefrontaldependent cognition. Neuropsychologia.

Dietrich, A., Taylor, J. T., \& Passmore, C. E. (2001). AVP (4-8) improves concept learning in PFC-damaged but not hippocampal-damaged rats. Brain Research, 919, 41-47.

Dixon, M., \& Laurence, J. R. (1992). Hypnotic susceptibility and verbal automaticity: Automatic and strategic processing differences in the Stroop color-naming task. Journal of Abnormal Psychology, 101, 344-347.

Duncan, J., \& Owen, A. M. (2000). Common regions of the human frontal lobe recruited by diverse cognitive demands. Trends in Neuroscience, 23, 475-483.

Farrell, P. A., Gustafson, A. B., Morgan, W. P., \& Pert, C. B. (1987). Enkephalins, catecholamines, and psychological mood alterations: Effects of prolonged exercise. Medicine and Science in Sports and Exercise, 19, 347-353.

Faymonville, M. E., Laureys, S., Degueldre, C., Del Fiore, G., Luxen, A., Frank, G., Lamy, M., \& Maquet, P. (2000). Neural mechanisms of antinocioceptive effects of hypnosis. Anesthesiology, 92, 1257-1267.

Frith, C. D., \& Dolan, R. (1996). The role of the prefrontal cortex in higher cognitive functions. Cognitive Brain Research, 5, 175-181.

Frith, C. D., \& Frith, U. (2001). Cognitive psychology —interacting minds - a biological basis. Science, 286, 1692-1695.

Fuster, J. M. (1995). Temporal processing-Structure and function of the human prefrontal cortex. Annals of the New York Academy of Sciences, 769, 173-181.

Fuster, J. M. (2000). Executive frontal functions. Experimental Brain Research, 133, 6670.

Gazzaniga, S. M., Ivry, R. B., \& Mangun, G. R. (1998). Cognitive neuroscience. New York: W.W. Norton.

Gilbert, P. F. C. (2001). An outline of brain function. Cognitive Brain Research, 12, 6174.

Glenister, D. (1996). Exercise and mental health: A review. Journal of the Royal Society of Health, 116, 7-13.

Gold, S. R., Gold, R. G., \& Milner, J. S. (1987). Daydreaming and mental health. Imagination Cognition and Personality, 6, 67-73.

Goldman-Rakic, P. S. (1992). Working memory and the mind. Scientific American, 261, 111117.

Ground, M., Pawlik, G., Walter, H., Lesch, O. M., \& Heiss, W.-D. (1995). Hypnotic cataplexy-induced changes of regional cerebral glucose metabolism. Psychiatry Research: Neuroimaging, 61, 173-179.

Gruzelier, J. H. (2000). Redefining hypnosis: Theory, methods and integration. Contemporary Hypnosis, 17, 51-70.

Hardcastle, V. G. (1999). The myth of pain. Cambridge, MA: MIT Press.

Hartman, E. (1966). The psychophysiology of free will. In R. Lowenstein, L. Newman, \& A. Solnit (Eds.), Psychoanalysis: A general psychology. New York: International University Press.

Hebb, D. O. (1939). Intelligence in man after large removal of cerebral tissue: Report of four left frontal lobe cases. Journal of General Psychology, 21, 73-87. 
Hegadoren, K. M., Baker, G. B., \& Bourin, M. (1999). 3,4-Methylenedioxy analogues of amphetamine: Defining the risks to humans. Neuroscience and Biobehavioral Reviews, 23, 539-553.

Herzog, H., Lele, V. R., Kuwert, T., Langen, K.-J., Kops, E. R., \& Feinendengen, L. E. (1990). Changed pattern of regional glucose metabolism during yoga meditative relaxation. Neuropsychobiology, 23, 182-187.

Hilgard, E. R. (1980). Consciousness in contemporary psychology. Annual Review of Psychology, 31, 1-26.

Hobson, J. A. (1988). The dreaming brain. New York: Basic Books.

Hobson, J. A. (2001). The dream drugstore. Cambridge, MA: MIT Press.

Hobson, J. A., \& McCarley, R. (1977). The brain as a dream-state generator: An activationsynthesis hypothesis of the dream process. American Journal of Psychiatry, 134, 1335-1348.

Hobson, J. A., Pace-Schott, E. F., \& Stickhold, R. (2000). Dreaming and the brain: Toward a cognitive neuroscience of conscious states. Behavioural and Brain Sciences, 23, 793-866.

Hoffman, P. (1997). The endorphin hypothesis. In W. P. Morgan (Ed.), Physical activity \& mental health (pp. 161-177). Washington: Taylor \& Francis.

Houde, O., \& Joyes, C. (1995). Logicomathematical development, prefrontal cortex, and inhibition-An example of categorization. Revue De Neuropsychologie, 5, 281-307.

Ide, K., \& Secher, N. H. (2000). Cerebral blood flow and metabolism during exercise. Progress in Neurobiology, 61, 397-414.

Jevning, R., Wallace, R. K., \& Beidebach, M. (1992). The physiology of meditation: A review. A wakeful hypometabolic integrated response. Neuroscience and Biobehavioral Reviews, 16, 415-424.

Kaiser, J., Barker, R., Haenschel, C., Baldeweg, T., \& Gruzelier, J. H. (1997). Hypnosis and event-related potential correlates of error processing in a Stroop-type paradigm: A test of the frontal hypothesis. International Journal of Psychophysiology, 27, 215-222.

Kalant, H. (2001). The pharmacology and toxicology of "ecstasy" (MDMA) and related drugs. Canadian Medical Association Journal, 165, 917-928.

Kallio, A., Revonsuo, A., Hämäläinen, H., Markela, J., \& Gruzelier, J. (2001). Anterior brain functions and hypnosis: A test of the frontal hypothesis. International Journal of Clinical and Experimental Hypnosis, 49, 95-108.

Keenan, J. P., Wheeler, M. A., Gallup, G. G., \& Pascual-Leone, A. (2000). Self-recognition and the right prefrontal cortex. Trends in Cognitive Sciences, 4, 338-344.

Kihlstrom, J. F. (1985). Hypnosis. Annual Review of Psychology, 36, 385-418.

Kirsch, I., \& Lynn, S. J. (1998). Social-cognitive alternatives to dissociation theories of hypnotic involuntariness. Review of General Psychology, 2, 66-80.

Knight, R. T., \& Grabowecky, M. (1999). Prefrontal cortex, time, and consciousness. In M. S. Gazzaniga (Ed.), The cognitive neurosciences (2nd ed., pp. 1319-1337). Cambridge, MA: MIT Press.

Kolb, B. (1984). Functions of the prefrontal cortex in the rat: A comparative view. Brain Research Review, 8, 65-98.

Kubitz, K. A., \& Pothakos, K. (1997). Does aerobic exercise decrease brain activation? Journal of Sport \& Exercise Psychology, 19, 291-301.

LaBerge, S. (1985). Lucid dreaming. Los Angels: Jeremy P. Tarcher.

Lazar, S. W., Bush, G., Gollub, R. L., Fricchione, G. L., Khalsa, G., \& Benson, H. (2000). Functional brain mapping of the relaxation response and meditation. NeuroReport, 11, $1581-1585$.

LeDoux, J. (1996). The emotional brain. New York: Touchstone.

Lhermitte, F. (1983). "Utilization behaviour" and its relation to lesions of the frontal lobes. Brain, 106, 237-255. 
Lhermitte, F., Pillon, B., \& Serdaru, M. (1986). Human autonomy and the frontal lobes. Part I: Imitation and utilization behavior: A neuropsychological study of 75 patients. Annals of Neurology, 19, 326-334.

Llinás, R. R., \& Paré, D. (1991). Of dreaming and wakefulness. Neuroscience, 44, 521-535.

Loeber, R. T., \& Yurgelun-Todd, D. A. (1999). Human neuroimaging of acute and chronic marijuana use: Implications for frontocerebellar dysfunction. Human Psychopharmacology, 14, 291-304.

Lou, H. C., Kjaer, T. W., Friberg, L., Wildschiodts, G., Holm, S. \& Nowak, M. (1999). A ${ }^{15} \mathrm{O}-\mathrm{H}_{2} \mathrm{O}$ PET study of meditation and the resting state of normal consciousness. Human Brain Mapping, 7, 98-105.

Mandell, A. J. (1981). The second wind. In M. H. Sacks \& M. L. Sachs (Eds.), Psychology of running (pp. 221-223). Champaign, IL: Human Kinetics.

Maquet, P., Peters, J. M., Aerts, J., Delfiore, G., Degueldre, C., Luxen, A., \& Franck, G. (1996). Functional neuroanatomy of human rapid-eye-movement sleep and dreaming. Nature, 383, 163-166.

Maquet, P., Faymonville, M. E., Degueldre, C., Delfiore, G., Frank, G., Luxen, A., \& Lamy, M. (1999). Functional neuroanatomy of hypnotic state. Biological Psychiatry, 45, 327-333.

Markowitsch, H. J. (1995). Cerebral bases of consciousness: A historical view. Neuropsychologia, 33, 1181-1192.

Mayberg, H. S. (1997). Limbic-cortical dysregulation: A proposed model of depression. Journal of Neuropsychiatry and Clinical Neuroscience, 9, 471-481.

Mishkin, M., Malamut, B., \& Bachevalier, J. (1984). Memory and habit: Two neural systems. In G. Lynch, J. J. McGaugh, \& N. M. Weinberger (Eds.), Neurobiology of learning and memory (pp. 66-77). New York: Guilford Press.

Newberg, A. B., Alavi, A., Baime, M., Pourdehnad, M., Santanna, J., \& d'Aquili, E. G. (2001). The measurement of regional cerebral blood flow during the cognitive task of meditation: A preliminary SECT study. Psychiatry Research Neuroimaging Section, 106, $113-122$.

Nofzinger, E. A., Mintun, M. A., Wiseman, M. B., Kupfer, D. J., \& Moore, R. Y. (1997). Forebrain activation in REM sleep: An FDG PET study. Brain Research, 770, 192-201.

Nordby, H., Hugdahl, K., Jasiukaitis, P., \& Spiegel, D. (1999). Effects of hypnotizability on performance of a Stroop task and event-related potential. Perceptual and Motor Skills, 88, 819-830.

Norman, D. A., \& Shallice, T. (1986). Attention to action: Willed and automatic control of behavior. In R. S. Davidson, G. E. Schwartz, \& D. Shapiro (Eds.), Consciousness and selfregulation (pp. 1-18). New York: Plenum.

Orne, M. T. (1959). The nature of hypnosis: Artifact and essence. Journal of Abnormal and Social Psychology, 58, 277-299.

Pargman, D., \& Baker, M. (1980). Running high: Enkephalin indicted. Journal of Drug Issues, $10,341-349$.

Petrides, M., \& Pandya, D. N. (1999). Dorsolateral prefrontal cortex: Comparative cytoarchitectonic analysis in the human and the macaque brain. European Journal of Neuroscience, 11, 1011-1036.

Pinker, S. (1999). How the mind works. New York: W.W. Norton.

Pistis, M., Porcu, G., Melis, M., Diana, M., \& Gessa, G. L. (2001). Effects of cannabinoids on prefrontal neuronal responses to ventral tegmental area stimulation. European Journal of Neuroscience, 14, 96-102.

Plotnick, A. B., Payne, P. A., \& O’Grady, D. J. (1991). Correlates of hypnotizability in children-Absorption, vividness of imagery, fantasy play, and social desirability. American Journal of Clinical Hypnosis, 34, 51-58. 
Pollen, D. (1999). On the neural correlate of visual perception. Cerebral Cortex, 9, 4-19.

Posner, M. (1994). Attention: The mechanism of consciousness. Proceedings of the National Academy of Sciences, 91, 7398-7403.

Povinelli, D. J., \& Preuss, T. M. (1995). Theory of mind: Evolutionary history of a cognitive specialization. Trends in Neuroscience, 18, 418-424.

Rainville, P., Hofbauer, R. K., Paus, T., Duncan, G. H., Bushnell, M. C., \& Price, D. D. (1999). Cerebral mechanisms of hypnotic induction and suggestion. Journal of Cognitive Neuroscience, 11, 110-125.

Ray, W. J., \& Faith, M. (1995). Dissociate experiences in a college age population: Follow-up with 1190 subjects. Personality and Individual Differences, 18, 223-230.

Rechtschaffen, A. (1978). The single-mindedness and isolation of dreams. Sleep, 1, $97-$ 109.

Ruben, R. T., \& Harris, G. J. (1999). Obsessive-compulsive disorders and the frontal lobes. In B. L. Miller \& J. L. Cummings (Eds.), The human frontal lobes: Functions and disorders (pp. 247-260). New York: Guilford Press.

Rylander, G. (1948). Personality analysis before and after frontal lobotomy. Research Publication-Association for Research in Nervous and Mental Disease, 27, 691-705.

Salmon, P. (2001). Effects of physical exercise on anxiety, depression, and sensitivity to stress: A unifying theory. Clinical Psychological Review, 21, 33-61.

Sarter, M., Givens, B., \& Bruno, J. P. (2001). The cognitive neuroscience of sustained attention: Where top-down meets bottom-up. Brain Research Reviews, 35, 146-160.

Scully, D., Kremer, J., Meade, M. M., Graham, R., \& Dudgeon, K. (1998). Physical exercise and psychological well being: A critical review. British Journal of Sports Medicine, 32, 111120.

Shallice, T., \& Burgess, W. (1991). Deficits in strategy application following frontal lobe damage in man. Brain, 114, 727-741.

Sheehan, P. W., Donovan, P., \& MacLeod, C. M. (1988). Strategy manipulation and the Stroop effect in hypnosis. Journal of Abnormal Psychology, 97, 455-460.

Siegel, R. K. (1985). LSD hallucination: From ergot to electric Kool-Aid. Journal of Psychoactive Drugs, 17, 247-256.

Singer, J. L. (1975). Navigating the stream of consciousness: Research in daydreaming and related inner experiences. American Psychologist, 30, 727-738.

Singer, J. L. (1978). Experimental studies of daydreaming and the stream of thought. In K. S. Pope \& J. L. Singer (Eds.), The stream of consciousness: Scientific investigations into the flow of human experience (pp. 209-227). New York: Plenum.

Singer, J., \& Pope, K. S. (1981). Daydreaming and imagery skills as predisposing capacities for self-hypnosis. International Journal of Clinical and Experimental Hypnosis, 29, 271-281.

Spanos, N. P. (1994). Multiple identity enactment and multiple personality disorder: A sociocognitive perspective. Psychological Bulletin, 116, 143-165.

Starkstein, S. E., \& Robinson, R. G. (1999). Depression and frontal lobe disorders. In B. L. Miller \& J. L. Cummings (Eds.), The human frontal lobes: Functions and disorders (pp. 247260). New York: Guilford Press.

Stone, V. E., Baron-Cohen, S., \& Knight, R. T. (1998). Frontal lobe contributions to the theory of mind. Journal of Cognitive Neuroscience, 10, 640-656.

Tart, C. T. (1979). Measuring the depth of an altered state of consciousness, with particular reference to self-report scales of hypnotic depth. In E. Fromm \& R. E. Shor (Eds.), Hypnosis: Developments in research and new perspectives (2nd ed.). New York: Aldine.

Taylor, J. G. (2001). The central role of the parietal lobes in consciousness. Consciousness and Cognition, 10, 379-417.

Vertosick, F. T. (2000). Why we hurt: A natural history of pain. San Diego: Hartcourt Brace. 
Vissing, J., Anderson, M., \& Diemer, N. H. (1996). Exercise-induced changes in local cerebral glucose utilization in the rat. Journal of Cerebral Blood Flow and Metabolism, 16, 729-736. Vogeley, K., Bussfeld, P., Newen, A., Herrman, S., Happe, F., Falkai, P., Maier, W., Shah, N. J., Fink, G. R., \& Zilles, K. (2001). Mind reading: Neural mechanisms of theory of mind and self-perspective. Neuroimage, 14, 170-181.

Vogeley, K., Kurthen, M., Falkai, P., \& Maier, W. (1999). Essential functions of the human self model are implemented in the prefrontal cortex. Consciousness and Cognition, 8, 343363.

Wik, G., Fischer, H., Bragee, B., Finer, B., \& Frederikson, M. (1999). Functional anatomy of hypnotic analgesia: A PET study of patients with fibromyalgia. European Journal of Pain: London, 3, 7-12. 